\title{
SOCIAL LEARNING WITHIN ELECTRONIC ENVIRONMENTS
}

\author{
Current perspectives and future directions
}

\author{
Carolyn Dowling \\ Australian Catholic University, 115 Victoria Parade, Fitzroy, Victoria 3165, Australia \\ c.dowling@patrick.acu.edu.au
}

\begin{abstract}
Within both educational institutions and the workplace, there are strong imperatives for learning to take place within an identifiably 'social' context, characterised by interactivity between learners and teachers, and between learners and their peers. Not only is this accepted as good pedagogical practice, but it is also important in situating learning within the type of cooperative, collaborative problem-solving environment characteristic of most contemporary workplaces. At the same time, online learning is becoming an increasingly important educational option for students at all stages of life. This paper explores some of the issues relating to how appropriate social modes of learning can be achieved within electronic environments, including consideration of the current and future roles of socially interactive software agents.
\end{abstract}

Key words: social learning, software agents, computer-mediated communication

\section{INTRODUCTION}

Within both formal educational institutions and the workplace, the idea, stemming most obviously from the work of Vygotsky (1978), that knowledge is 'socially constructed' through a process of negotiation between learners and the world around them, has achieved wide acceptance in recent years. The notion that learning should be characterised by interaction not just between learners and their teachers but also between learners and their peers, is now regarded by most educators as good pedagogical practice. It is also seen as reflecting the way in which 
knowledge is typically acquired and used in the team-oriented environment of the contemporary workplace.

At the same time, learning online is becoming an increasingly important option for full time, part time and even 'occasional' students. Wellrecognised advantages include the ability to study at 'any place' and at 'any time'. This flexibility is of benefit not just to full time workers, but also to those students who are obliged to mix work with study in order to survive financially, or who may have difficulty in physically attending a particular educational institution. A further aspect of online learning that contributes to its attractiveness, particularly to younger people, is its synergy with the way in which they utilise Information and Communication Technologies to conduct much of their work and their leisure activities. But how effective are online educational environments in providing the social interactivity that we currently believe characterises the best pedagogical practices, and how can we ensure that we maximise this aspect of electronic learning?

\section{CURRENT MODES OF ENCOURAGING SOCIAL INTERACTIVITY ONLINE}

Today a great deal of online learning is conducted through the medium of software 'shells' which enable teachers or instructors with little expertise to insert their course materials into predetermined structures. Most of these products include provision for course schedules, course materials, links to appropriate websites, testing of students' skills and knowledge, different types of record keeping and, very importantly, a range of options for facilitating communication. The types of communication facilitated would normally encompass teacher-to-student-group, teacher-to-individual-student, student-to-teacher and student-to-student, in both synchronous and asynchronous modes. It is customary to also distinguish between 'public' modes of interaction such as bulletin boards and contributions to email lists or 'open' chat environments, and 'private' one-on-one exchanges such as individually addressed email messages that clearly fulfil a different purpose.

How do these opportunities for person-to-person interaction compare with those available in the face-to-face classroom? Traditional classroom organisation favours the teacher-to-student-group model, with some provision for communication between the teacher and an individual student although, when this takes place within the public space of the physical classroom, it generally suffers from a lack of privacy and confidentiality. Traditional classrooms also incorporate some potential for student-to-teacher communication, although again this could rarely be classed as 'private' or individual communication in the usual sense. While student-to-student 
communication is facilitated in many face-to-face classrooms, often through organisational structures such as 'group work', there are generally severe limitations imposed on the time and place at which this type of interaction is permitted. Opportunities for unmandated discussion between students do occur, but often the necessarily furtive nature of such communication diminishes its potential contribution to learning.

By contrast, the variety of modes of communication available within most online learning environments provides the flexibility for a range of different types of interaction. In a recent evaluation of courses offered in a combination of face-to-face and online modes, it was noted by this author that students and teachers like reported that they appreciated the ease with which communication could be facilitated both privately between individuals and between members of different sub-groups of the class identified for particular purposes (Student course evaluations, 2002).

Computer mediated social interactions have other potential advantages in relation to learning. In the absence of social 'markers' such as physical appearance, dress and so on, students may feel more able to engage in interaction than would otherwise be the case. Particularly where the course takes place entirely online, this may be enhanced by the degree to which they are able to exercise positive control over the persona they present to the rest of the group.

In summary, it would appear that the electronic medium has some advantages in facilitating the types of social interaction that are believed to mediate effective learning. For both student and teacher there are enhanced opportunities for individualised, highly specific interactions, in addition to an increased freedom to communicate at times of their own choosing, and perhaps at greater length and in more detail than is possible in a physical setting.

It is important to acknowledge, however, that currently this type of communication is overwhelmingly textual in nature, and lacks the richness of face-to-face interaction where words are reinforced by aspects of physical presence such as gesture, tone of voice, facial expression, 'body language' and so on. In the face-to-face classroom students are 'known' both to one another and to teachers in a very different sense. It is well recognised that electronically mediated communication is not 'the same' as face-to-face (Dowling, 2000), and this should be taken into account when considering the advantages and disadvantages of online learning. 


\section{FUTURE DIRECTIONS}

Given that, in addition to their other advantages, online learning environments do have special potential for facilitating certain types of pedagogically helpful social interactions, how might we expect this aspect of their design to develop in the future?

More extreme facilitation of 'any place, any time' learning might well occur not through the traditional computer interface, but through the medium of portable handheld devices. Many of these already have the ability to combine auditory and visual data, including video representations of the speaker, with textual information. Furthermore they operate in a wireless environment, unencumbered by the need to be 'plugged in' (Multisilta, 2003). While traditionalists might balk at the idea of delivering instruction by means of a device that permits such a small amount of information to be visible at a time, it should be acknowledged that many young people now conduct much of the important 'business' of their lives through the medium of text messaging. As reported recently, 'More than 40 per cent of people aged 15 or older in Europe's three biggest economies use short messaging service (SMS) on cellular handsets, while 30 per cent use computer email, says research company GartnerG2' (McLuskey, 2002). The recent expansion of Instant Messaging (IM) introduces a further element with the potential to change the way in which we interact for work, leisure and also study.

In relation to the limitations posed by the screen dimensions of hand-held technologies, it should be remembered that in the 1980s the introduction of hypertext, now familiar to us as one of the structural foundations of the World Wide Web, caused considerable concern to theorists and researchers because of the need to 'chunk' information into segments significantly smaller than those to which we were accustomed in the context of paperbased print media. In this as in so many other aspects of our lives, we have proved to be extremely adaptable. Perhaps of greater concern are the broader social consequences of constant availability undermining the ability of individuals to structure the different aspects of their lives according to their personal preferences. While there is certainly enhanced potential for integrating learning activities within an environment appropriate to an individual learner, such as a workplace, there is also the possibility of educational activities spilling over into what is normally regarded as leisure time to a degree which would be unacceptable to many people.

Another area rich in potential for encouraging interactivity within online learning environments is the use of software agents, computer programs possessing varying degrees of 'intelligence', autonomy and personification, as participants in the social interactions that mediate learning (Dowling, 2002). The roles that can be undertaken by these electronic constructs are 
rich and varied. Many of them, such as information retrieval and record keeping, incorporate a minimal 'social' component at best. Others, however, are specifically designed so as to enhance both the quantity and the quality of the interactions taking place within the online environment. Johnson (1998) provides the following overview of the possible roles of such entities, closing with specific reference to their capacity to contribute to the 'social' aspects of knowledge construction.

"Pedagogical agents are autonomous agents that support human learning, by interacting with students in the context of interactive learning environments. They extend and improve upon previous work on intelligent tutoring systems in a number of ways. They adapt their behaviour to the dynamic state of the learning environment, taking advantage of learning opportunities as they arise. They can support collaborative learning as well as individualized learning, because multiple students and agents can interact in a shared environment. Given a suitably rich user interface, pedagogical agents are capable of a wide spectrum of instructionally effective interactions with students, including multimodal dialog. Animated pedagogical agents can promote student motivation and engagement, and engender affective as well as cognitive responses" (Johnson, 1998).

The most obvious classroom roles that incorporate a social dimension are those of the teacher or tutor, and the fellow learner. Within the latter category we can include what is sometimes to referred to as the 'tutee' someone to whom the learner 'teaches' material that he or she has learned. This is an important aspect of the mutuality that characterises social learning.

While the idea of a computer program 'teaching' a student may appear to represent a return to the bad old days of overly simplistic instructional software, Johnson is correct in indicating that today's software agents are far more complex and flexible in their capabilities and modes of operation. For example:

"The user mental model of the system should be based on the metaphor of the 'invited professor' rather than the 'knowing everything own tutor'. ... Our first findings confirm the observation that today's users, accustomed to hypertext-like interaction, are more likely to accept this collaborative teaching metaphor, according to which their tutoring system is viewed as an intelligent hypertext browser, offering links to other tutoring systems with the right content and at the right time" (Solomos \& Avouris, 1999).

The contemporary image of the teacher as a facilitator of learning rather than as the 'sage on the stage' is further reflected in descriptions of agent roles such as: "Each student working on the project will have an agent, operating in the background, watching progress, measuring it against the 
plan, and taking remedial action when necessary" (Whatle, Staniford, Beer, \& Scown, 1999),

The notion of agents as fellow learners is most readily exemplified by the work of Chan and his colleagues over a number of years (Chan, 1996; 1998). As is the case with human classmates, their 'learning companions', anthropomorphised for young students in the form of friendly animals, possess differing competencies and degrees of knowledge of the content or skills domain being studied. A further example is described by Ju (1998) who writes of a computer based peer tutoring system employing two categories of agent - an 'expert', and a 'learner':

“... students become active learners who are guided to learn by teaching a computer. After the students watch how the computer expert solves a set of linear equations [the program] helps the human student act as a teacher in order to learn more about the subject matter. At this time, the computer plays the role of a student ..." (Ju, 1998).

While many educators would be uncomfortable with the notion that interaction with an electronic entity should entirely replace human-to-human communication in the classroom, it is easier to be supportive of pedagogical agents if they are seen as fulfilling a complementary rather than a substituting role within the online community of learners. Appropriate actions might include providing immediate and highly specific feedback to students at times when a human respondent is not readily available. The 'any time' aspect of electronic learning can and often does place extraordinary demands on human teachers in terms of their availability to provide support to their students at pedagogically appropriate moments.

Of course as with all aspects of electronic learning environments, there is always some potential for features, including socially interactive software agents, to be developed 'because we are able to', rather than because they will necessarily contribute positively to student learning. Happily this is generally well recognised by developers, as exemplified in the following comment made about one online learning environment based on constructivist principles, emphasising communication and collaboration, and also featuring elements of advanced technology:

"The author does not recommend that all CMC systems should have virtualreality quality or avatars built-in. These tools can be made available and customized by users. The freedom of choices empowers the users to take control of their learning process. In some instances, the add-on tools can be a distraction for online conversation. In some instances they are great tools for increasing interpersonal connections. The use of the tools should match the pedagogical goals of an instructional unit, not just used for the sake of using the tools" (Chou, 2001). 


\section{CONCLUSION}

The conjunction of two important imperatives in contemporary education and training is creating both opportunities and dilemmas for teachers and their students. On the one hand, new developments in mobile communication technologies suggest exciting and challenging possibilities for further enhancing the provision of opportunities for learning at a time and place of the learner's (or their employer's) own choosing. At the same time, there is increased recognition of the importance of learning taking place within a social context that reflects and models the type of cooperative, collaborative problem-solving environment characteristic of the modern workplace. Central to the productive resolution of what might appear at first to be somewhat contradictory trends in education is the capacity for electronically mediated learning environments to provide opportunities for social interaction in the service of knowledge construction. New and developing technologies provide a very flexible range of possibilities for communication between teachers and learners, between learners and their peers, and between human learners and pedagogical software agents. Interestingly, there are certain features of the newer mobile technologies that might have the potential to enhance the apparent 'social' abilities of interactive agents. In addition to augmenting the 'presence' of human communicators, the video and multimedia capabilities of handheld devices could be utilised to add depth to the animation or personification of agent software. A further area for investigation is the degree to which the abbreviated forms of text best suited to the small screen might assist in blurring the distinction between human and non-human participants in the social interactions mediating learning. It summary, it can be argued that significant synergies may exist between the new directions in communications technologies and the capacity of agent software to enhance the level of timely and informative communication within electronically mediated learning environments.

\section{REFERENCES}

Chan, T. W. (1996). Learning companion systems, social learning systems, and the global social learning club, Journal of Artificial Intelligence in Education. 7(2).

Chan, T. W. (1998). The past, present, and future of educational agents. Retrieved 03/02/2000 from http://www.apc.src.ncu.edu.tw/apc/ppt_chan.html.

Chou, C. (2001). Formative Evaluation of Synchronous CMC Systems for Learner-Centred Online Course. Journal of Interactive Learning Research, 12(2/3), 173-192. 
Dowling, C. (2000). Social Interactions and the Construction of Knowledge within Computer Mediated Learning Environments. In T. Downes. \& D. Watson (Eds.), Learning in a Networked Society. (pp. 165-174). Kluwer, Boston.

Dowling, C. (2002). The Socially Interactive Pedagogical Agent within Online Learning Communities, Proceedings of the International Conference on Computers in Education ICCE 2002, (pp. 30-34). Auckland, New Zealand.

Johnson, W.L., (1998). Pedagogical Agents. Proceedings of, the Sixth International Conference on Computers in Education (ICCE '98), (pp.13-22). Beijing, China, October 14-17.

Ju, Y. (1998), Development and formative evaluation of a computer-based peer tutoring system. Proceedings of, the Sixth International Conference on Computers in Education (ICCE '98), (pp. 559-566). Beijing, China, October 14-17.

McLuskey, D. (2002), SMS eats into email in Europe, The Australian (IT section), 12.11.2002, p.6.

Multisilta, J. (2003), The Teacher in the Mobile World, ICT and the Teacher of the Future, (pp.257-266). Kluwer, Boston.

Solomos, K., \& Avouris, N. (1999). Learning from Multiple Collaborating Intelligent Tutors: An Agent-Based Approach. Journal of Interactive Learning Research, 10(3/4) 243-263.

Dowling, C. (2002). Student course evaluations, June 2002.

Vygotsky, L. (1978), Mind in Society, Cambridge, MA: Harvard University Press.

Whatley, J., Staniford, G, Beer, M. \&Scown, P. (1999), Intelligent Agents to Support Students Working in Groups Online. Journal of Interactive Learning Research.10, (3/4), 235-243.

\section{BIOGRAPHY}

Carolyn Dowling is currently Head of the School of Business and Informatics at Australian Catholic University. During the last twenty years she has taught and researched a number of different aspects of ICT at University level. Her current research is focussed on electronically mediated communication and agent technologies. 